\title{
Successful intrauterine treatment of nonimmune hydrops fetalis due to pericardial tumor
}

\author{
Aytul CORBACIOGLU ESMER @ , Helen BORNAUN @, Isil TURAN BAKIRCI
}

\begin{abstract}
The prenatal diagnosis of pericardial tumor is extremely rare. It may have serious consequences such as massive pericardial effusion, cardiac tamponade, hydrops fetalis and fetal demise. We present a case of nonimmune hydrops fetalis secondary to pericardial mass which was successfully treated in utero by serial pericardiocentesis. Also, we present a review of the literature regarding the intrauterine interventions performed in pericardial tumor cases. At 24 weeks' gestation ascites, diffuse subcutaneous edema at the thorax and abdomen, scalp edema and two masses within severe pericardial effusion were detected. After the pericardiocentesis performed at 25, 28 and 29 weeks' gestation, ascites and scalp edema resolved, and the pericardial effusion regressed gradually. The size of masses did not change throughout the gestation. After the infant was born at 37 weeks' gestation, the masses shrunk spontaneously within five months.

Keywords: Pericardial tumor, Pericardiocentesis, Intrauterine treatment, Nonimmune hydrops fetalis, Pericardial effusion
\end{abstract}

\section{Introduction}

Cardiac tumors are rarely seen in fetus with an incidence of $0.14 \%$ [1]. The vast majority of these tumors are rhabdomyoma, teratoma and fibroma, whereas yolk sac tumor, multicystic hamartoma, hemangioma, lymphangioma, rhabdomyosarcoma, fibrosarcoma and epicardial lipoma are rare histologic types [2]. In addition,

Aytul Corbacioglu Esmer (ヌ), Isil Turan Bakirci

Division of Maternal Fetal Medicine, Department of Obstetrics and Gynecology, Istanbul Kanuni Sultan Suleyman Research and Teaching Hospital, Istanbul, Turkey.

e-mail: aytulcorbacioglu@gmail.com

Helen Bornaun

Division of Pediatric Cardiology, Department of Pediatrics, Istanbul Kanuni Sultan Suleyman Research and Teaching, Istanbul, Turkey pericardial cavity is an extremely rare location, and intrapericardial masses may have severe consequences such as massive pericardial effusion, cardiac tamponade, hydrops fetalis and fetal demise [3-8]. In the literature, there are only a few reports of prenatal therapy, such as pericardiocentesis, pericardioamniotic shunt and thoracoamniotic shunt in order to prevent tamponade, to resolve hydrops fetalis and to delay the delivery $[4,7,9,10]$.

We would like to present a case of nonimmune hydrops fetalis due to pericardial masses treated succesfully with serial pericardiocentesis.

\section{Case Report}

A 33-year-old woman, gravida 2, para 1, was referred to our clinic at 24 weeks' gestation due to hydrops fetalis. Detailed sonography revealed ascites, diffuse subcutanaeous edema at the thorax and abdomen, scalp edema and severe pericardial effusion. Echocardiography showed two masses located within the pericardial effusion; an echogenic mass of $19 \mathrm{x}$ $9.2 \mathrm{~mm}$ originating from left ventricular wall, and a mass of 10 × $6 \mathrm{~mm}$ with solid and cystic components arising from right ventricular free wall (Figure 1). Ventricular inflow and outfow tracts were normal and no other anomalies were detected. Middle cerebral artery peak systolic velocity was in the normal levels. Indirect Coombs test and maternal toxoplasmosis, rubella, cytomegalovirus, and herpes simplex (TORCH) serology screens were negative. The differential diagnosis included pericardial teratoma or hemangioma, and nonimmune hydrops fetalis was thought to be secondary to the severe pericardial effusion, since it filled the thorax almost completely. Prenatal magnetic resonance imaging (MRI) did not add further information. At 25 weeks' gestation after two doses of bethamethasone 
injection, $25 \mathrm{~mL}$ serohemorrhagic fluid was drained using 20 -gauge needle under sonographic guidance, and fetal blood sampling for karyotyping was performed. Toxoplasma and cytomegalovirus (CMV) polymerase chain reaction (PCR) findings were negative, and fetal karyotype was normal. The cytology of the fluid revealed abundant erythrocytes and leucocytes. Since, the severe effusion reaccumulated one week later, a second pericardiocentesis was performed, and $20 \mathrm{~mL}$ serohemorrhagic fluid was drained. At 28 weeks' gestation as the ascites and scalp edema were persisting, the patient underwent another pericardiocentesis yielding 30 $\mathrm{mL}$ serohemorrhagic fluid. At 29 weeks' gestation, ascites and scalp edema resolved and only a mild pericardial effusion measuring $10 \mathrm{~mm}$ remained. In the following weeks, the effusion regressed slowly measuring $3 \mathrm{~mm}$ at 37 weeks' gestation. The masses were almost at the same size throughout the gestation.

At 34 weeks' gestation an additional asymmetric fetal growth restriction was detected. The amount of amniotic fluid and Doppler findings were normal. At 37 weeks' gestation, since cerebroplacental ratio was detected lower than $3^{\text {rd }}$ percentile, the decision of delivery was made. A 2390-gram male baby with an Apgar score of 5 and 9 at 1 and 5 minutes was delivered by a cesarean section due to the breech presentation. The infant was in good condition with stable vitals on physical examination. On chest auscultation, the heart beats were rhythmic, and there was good bilateral ventilation. There was a normal sinus rhythm on electrocardiogram. The chest radiograph showed normal cardiac silhouette. Transthoracic echocardiography revealed a structurally normal heart without a hemodynamically relevant pericardial effusion, and an intrapericardial tumor measuring $19 \times 6 \mathrm{~mm}$ in the left ventricular free wall that did not compromise blood flow into the cardiac cavities (Figure 2a). On the other hand, no significant mass was observed in the free wall of the right ventricle. Neither pericardiocentesis nor emergency cardiothoracic surgery was necessary. The diagnosis of an intrapericardial tumor was confirmed by cardiac computed tomography (CT), which showed a homogeneous, soft tissue density measuring $7 \times 14 \mathrm{~mm}$ located posterior to the left atrioventricular junction.

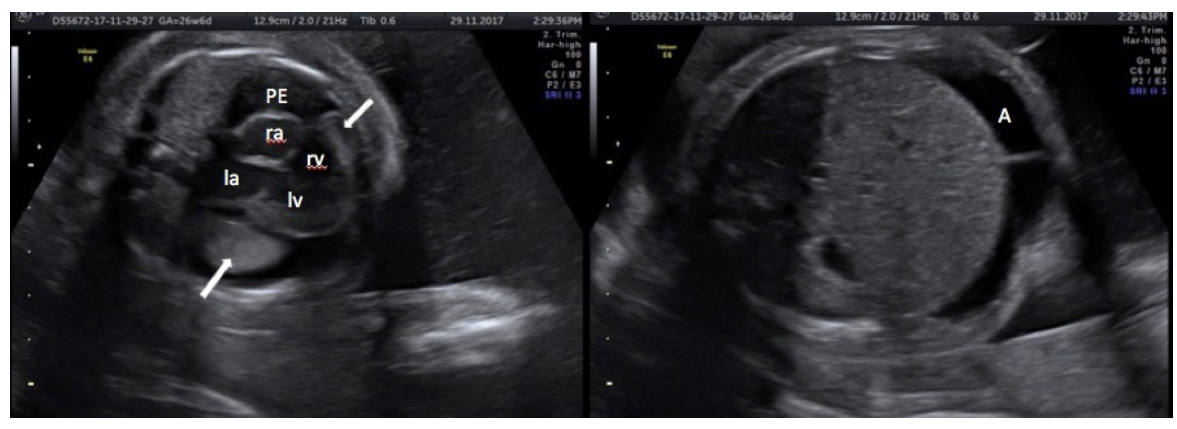

Figure 1. Ultrasound at 26 weeks' gestation demonstrating, the two pericardial masses (arrows), pericardial effusion, ascites and skin edema.

A, ascites; PE, pleural effusion; ra, right atrium; la, left atrium; rv, right ventricle; lv, left ventricle

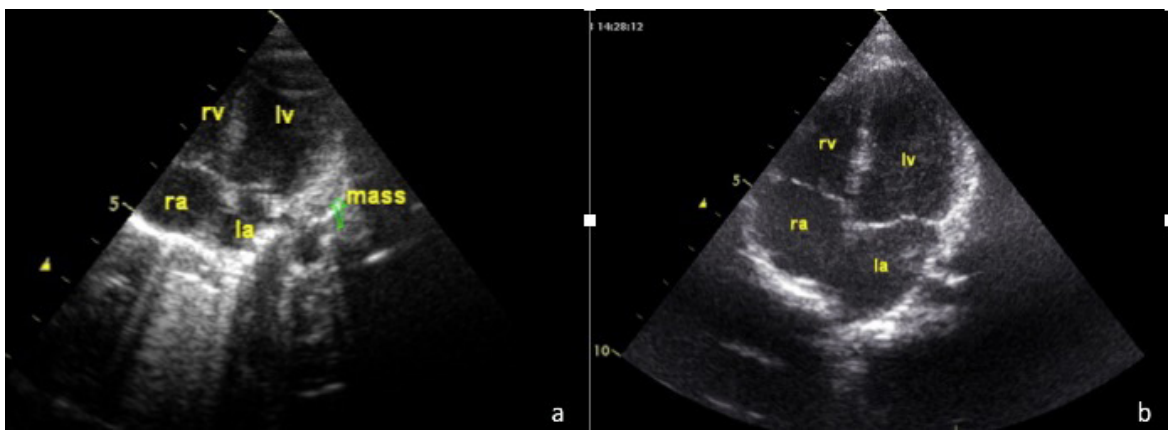

Figure 2. Postnatal echocardiography performed (a) immediately after birth demonstrating a pericardial mass arising from the left ventricular free wall (arrow) and (b) after 5 months demonstrating no obvious mass.

ra: right atrium, la:left atrium, rv: right ventricle, lv: left ventricle 
At the time of writing, our case was 5 months old. No complications were detected during follow-up. Serial echocardiography revealed gradual regression of the mass which shrunk completely within 5 months (Figure 2b).

\section{Discussion}

Teratoma comprises the vast majority of pericardial tumors [2]. It is typically detected as an inhomogeneous mass with solid and cystic areas and sometimes with foci of calcification, located on the right anterior heart border associated with pericardial effusion [6]. Hemangioma is another tumor presenting with pericardial effusion, hydrops and tamponade $[2,11]$. Cutaneous hemangiomas can also be present in these cases. It is associated with a favorable outcome and tends to regress even without therapy [2] By contrast, teratomas are mostly large tumors and cause cardiovascular and respiratory distress in newborn requiring prompt surgical resection [12]. Also, mesothelioma and bronchogenic cysts may have intrapericadial location [13]. The differential diagnosis is not possible in utero, however, it is not important in the management of pregnancy. Since, the teratoma is the most common histologic type, we first considered that the masses were intrapericardial teratoma. However, as they resolved spontaneously, we now think hemangioma as the most possible histology.
Postnatal management include pericardiocentesis which may be necessary immediately after delivery [14]. When the patient is stabilized, cardiac surgery is usually performed due to the reaccumulation of pericardial fluid $[4,7,15,16]$. Alternatively, steroid therapy may be used for cardiac vascular tumors [11]. Unlike the previously reported cases, in our case pericardial effusion did not reaccumulate after birth, and the mass shrunk spontaneously. Therefore, there was no need for a surgical interventional.

The pericardial effusion secondary to pericardial tumors appears as a consequence of the rupture of cystic areas, or serous effusion from the surface of the tumor, or obstruction of cardiac and pericardial lymphatics. In utero pericardiocentesis relieves the obstruction of venous return. In the presented case, since the nature of pericardial effusion was hemorrhagic, we speculate that the hemorrhage of the tumor resulted in the pericardial effusion. The severe effusion rather than the mass effect of the tumor caused hydrops due to the compression of venous and lymphatic return. Therefore, the drainage of this fluid successfully resolved the hydrops fetalis. However, if the mass itself compresses the venous and lymphatic return, pericardiocentesis is not successful in restoring venous return. In this circumstances, the only option seems to be the fetal surgical resection in early hydrops cases. However, there is only scarce data regarding this procedure in the literature and it is highly

Table I. The intrauterine interventions performed in pericardial tumor cases

\begin{tabular}{|c|c|c|c|c|}
\hline Reference & Gestational age at intervention & Intervention & Indication & Outcome \\
\hline Benatar, 1992 (18) & 31 & $\mathrm{PC}$ & Large effusion & Alive \\
\hline Bruch, 1997 (3) & 25 & $\mathrm{PC}$ & Large effusion & Alive \\
\hline Paw, 1997 (12) & 24 & $\mathrm{PC}(\mathrm{x})$ & Tamponade, hydrops & Alive \\
\hline Sklansky, 1997 (4) & 20 & PC (2) & Tamponade & Alive \\
\hline Fujimori, 1999 (16) & 34 & $\mathrm{PC}$ & Hydrops & Alive \\
\hline Sepulveda, 2000 (15) & 37 & $\mathrm{PC}(2)$ & Large effusion & Alive \\
\hline Thorp, 2000 (19) & 34 & $\mathrm{PC}$ & Hydrops & Alive \\
\hline Sydorak, 2002 (17) & 18 & Fetal surgery & Hydrops & Neonatal death ${ }^{1}$ \\
\hline Laquay, 2003 (5) & 21 & PC & Hydrops & Alive \\
\hline Laquay, 2003 (5) & 28 & $\mathrm{PC}$ & Hydrops & Alive \\
\hline Tollens, 2003 (6) & 29 & $\mathrm{PC}$ & Hydrops & Alive \\
\hline Grebille, 2003 (7) & 28 & PC, TAS & Hydrops & Alive \\
\hline Kamil, 2006 (21) & 25 & $\mathrm{PC}(5)$ & Hydrops & Alive \\
\hline Bader, 2006 (9) & 23 & PC, PAS & Hydrops & Neonatal death ${ }^{2}$ \\
\hline Czernik, 2006 (22) & 24 & $\mathrm{PC}(3)$ & Hydrops & Alive \\
\hline Devliger, 2009 (10) & 24 & PC (2), PAS & Large effusion & Alive \\
\hline Rychik, 2016 (8) & 22 & $\mathrm{PC}$ & Large effusion & Intrauterine death \\
\hline Rcyhik, 2016 (8) & 22 & Fetal surgery & Ascites, large tumor & Alive \\
\hline Presented case & 24 & $\mathrm{PC}(3)$ & Hydrops & Alive \\
\hline
\end{tabular}

PC: Pericardiocentesis, PAS: Pericardio-amniotic shunting, TAS: Thoraco-amniotic shunting

$\mathrm{X}$ : Multiple times

${ }^{1}$ Preterm delivery at 27 weeks' gestation due to mirror syndrome

${ }^{2}$ Spontaneous preterm delivery at 25 weeks' gestation 
invasive [8, 17]. Table I summarizes the intrauterine interventions performed in pericardial tumor cases. Out of 18 cases published in the literature, two cases underwent open surgery [8, 17], two cases underwent pericardioamniotic shunting $[9,10]$ and one case underwent thoracoamniotic shunting following pericardiocentesis [7]. In others, single or serial pericardiocentheses were performed for hydrops or tamponade or large pericardial effusion [3, 4, $6,8,12,15,16,18-22]$. Only one intrauterine demise after open surgery [8], and two neonatal death due to preterm delivery following pericardiocentesis were reported $[9,17]$.

An algorithm for the management of the pericardial tumors has been proposed by Sydorak et al [17]. In case of hydrops fetalis, if the gestational age is less than 28 weeks, in utero pericardiocentesis is suitable for cystic masses with large effusion, while surgical resection is the only option for solid masses with small effusion. However, in cases beyond 28 weeks of gestation, if the lungs are mature, early delivery and postnatal surgery is recommended. If the lungs are immature, serial pericardiocentesis can be performed to delay the delivery until the pulmonary maturity. On the other hand, fetuses in whom hydrops does not occur may be safely followed up and treated postnatally [2].

In conclusion, pericardiocentesis is an important and lifesaving procedure in severe pericardial tumor cases, especially when there is a large pericardial effusion with a small mass.

\section{Disclosure statement}

The authors declare that there is no conflicts of interest.

\section{References}

1. Holley DG, Martin GR, BrennerJI. Diagnosis andmanagement of fetal cardiac tumors: a multicenter experience and review of published reports. J Am Coll Cardiol 1995;26:516-20. doi: 10.1016/0735-1097(95)80031-B.

2. Isaacs H. Fetal and neonatal cardiac tumors. Pediatr Cardiol 2004;25:252-73. doi:10.1007/s00246.003.0590-4.

3. Bruch SW, Adzick NS, Reiss R, et al. Prenatal therapy for pericardial teratomas. J Pediatr Surg 1997;32:1113-5. doi: 10.1016/ S0022-3468(97)90410-3.

4. Sklansky M, Greenberg M, Lucas V, et al. Intrapericardial teratoma in a twin fetus: Diagnosis and management. Obstet Gynecol 1997;89:807-9. doi:10.1016/S00297844(97)00031-8.

5. Laquay N, Ghazouani S, Vaccaroni L, et al. Intrapericardial teratoma in newborn babies. Eur J Cardiothorac Surg 2003;23:642-44. doi:10.1016/S1010-7940(02)00861-8.
6. Tollens T, Casselman F, Devligrt H, et al. Fetal cardiac tamponade due to an intrapericardial teratoma. Ann Thorac Surg 1998;66:559-60. doi:10.1016/S0003-4975(98)00508-6.

7. Grebille AG, Mitanchez D, Benachi A, et al. Pericardial teratoma complicated by hydrops: successful fetal therapy by thoracoamniotic shunting. Prenat Diagn 2003,23: 735-9. doi: 10.1002/pd.698.

8. Rychik J, Khalek N, Gaynor JW, et al. Fetal intrapericardial teratoma: natural history and management including successful in utero surgery. Am J Obstet Gynecol 2016;215: 780. e1-e7. doi:10.1016/j. ajog.2016.08.010.

9. Bader R, Hornberger LK, Nijmeh LJ, et al. Fetal pericardial teratoma: presentation of two cases and review of the literature. Am J Perinatol 2006;23:53-8. doi: 10.1055/s_-2005-923433.

10. Devliger R, Hindryckx A, Van Miegham T, et al. Therapy for foetal pericardial tumours: Survival following in utero shunting, and literature review. Fetal Diagn Ther 2009;25:407-12. doi:10.1159/000236156.

11. Riggs T, Sholl JS, Ilbawi M, et al. In utero diagnosis of pericardial tumor with successful surgical repair. Pediatr Cardiol 1984;5:23-6.

12. Paw PT, Jamieson SW. Surgical management of intraperciardial teratoma diagnosed in utero. Ann Thorac Surg 1997;64:552-4. doi:10.1016/S0003-4975(97)00585-7.

13. Todros T, Gaglioti P, Presbitero P. Management of a fetus with intrapericardial teratoma diagnosed in utero. J Ultrasound Med 1991;10:287-90. doi: 10.7863/jum.1991.10.5.287.

14. Perez-Aytes A, Sanchis N, Barbal A, et al. Non-immunological hydrops fetalis and intrapericardial teratoma: Case report and review. Prenat Diagn 1995;15:859-63. doi:10.1002/ pd.970150912.

15. Sepulveda W, Gomez E, Gutierrez J. Intrapericardial teratoma. Ultrasound Obstet Gynecol 2000;15:547-8. doi:10.1046/j.1469-0705.2000.00144.x

16. Fujimori K, Honda S, Akutsu H, et al. Prenatal diagnosis of intrapericardial teratoma: a case report. J Obstet Gynaecol Res 1999;25:133-6. doi:10.1111/j.1447-0756.1999.tb01135.x.

17. Sydorak RM, Kelly T, Feldstein VA, et al. Prenatal resection of o fetal pericardial teratoma. Fetal Diagn Ther 2002;17:2815. doi: 10.1159/000063180.

18. Benatar A, Vaughan J, Nicolini U, et al. Prenatal pericardiocenthesis: Its role in the management of intrapericardial teratoma. Obstet Gynecol 1992;79:856-9.

19. Thorp JA, Geidt A, Gelatt M, et al. Decompression of fetal cardiac tamponade caused by congenital capillary hemangioma of the pericardium. Obstet Gynecol 2000;96:816-7. doi:10.1016/S0029-7844(00)01045-0.

20. Laquay N, Ghazouani S, Vaccaroni L, et al. Intrapericardial teratoma in newborn babies. Eur J Cardiothorac Surg 2003;23:642-4. doi: 10.1016/S1010-7940(02)00861-8.

21. Kamil D, Geipel A, Schmitz C, et al. Fetal pericardial teratoma causing cardiac insufficiency: prenatal diagnosis and therapy. Ultrasound Obstet Gynecol 2006;28:972-8. doi: 10.1002/uog.3829.

22. Czernik C, Stiller B, Hübler M, et al. Hydrops fetalis caused by a large intrapericardial teratoma. Ultrasound Obstet Gynecol 2006;28:972-8. doi:10.1012/uog.3852. 